\title{
DINÂMICA DA ESTRATÉGIA LOGÍSTICA EM EMPRESAS BRASILEIRAS
}

RESUMO

O objetivo do artigo é analisar a estratégia logística das empresas brasileiras a partir da mensuração do impacto de diferentes características do negócio sobre as principais decisões de produção e distribuição física de produtos acabados. Foi conduzida uma pesquisa de campo em empresas listadas no Ranking Exame para confrontar as quatro políticas propostas por Pagh e Cooper (1998), derivadas das combinações entre centralizar/descentralizar os estoques e produzir para estoque/produzir contra-pedido, com diversas características do produto, da operação e da demanda. Técnicas de análise multivariada, como as análises de regressão logística simples e multinomial, foram empregadas, permitindo o desenvolvimento de diversos modelos quantitativos. Seus resultados apontam para avanços acadêmicos e gerenciais, como a quantificação e hierarquização de relações empíricas descritas na literatura e o direcionamento da atenção gerencial para as variáveis rel evantes à decisão. O s model os também permitem avaliar a adequação dessas políticas para diferentes valores do tempo de entrega, da variabilidade das vendas e do grau de obsolescência dos produtos.

\section{Peter Fernandes Wanke}

COPPEAD-UFRJ

\footnotetext{
ABSIRACT The article analyses the logistics strategy of Brazilian companies measuring the impact of business characteristics on production and distribution decisions. A survey of the companies ranked by the news magazine Exame was conducted to test the four policies advanced by Pagh and Cooper (1998) against several characteristics of products, operations and demand. The policies combineinventory centralization/decentralization and made to order/made to stock decisions. M odels were developed using multivariate techniques, including simple and multinomial regression. The results allowed the ranking and quantification of relationships previously found in the liter ature. These results might be useful when managers have to make logistics decisions.
}

PALAVRAS-CHAVE Logística, estratégia, produção, distribuição física, produtos acabados.

KEYWORDS Logistics, strategy, production, physical distribution, finished products. 


\section{INTRODUÇÃO}

Apesar do grande volume de artigos publicados anualmente sobre logística, uma pequena fração destina-se à análise da estratégia logística e de suas principais políticas. A maioria dessa pequena fração não se ocupa de estoques nem do fluxo de produtos, mas da utilização de "recursos como as tecnologias de informação, os modais de transporte e as redes de instalações" (Wanke, 2003, p. 2). Isso é paradoxal quando se considera que a logística é geralmente definida como "a parte do gerenciamento de cadeias de suprimento responsável pelo planejamento, implementação e controle do fluxo de produtos e de informações do ponto de origem ao de consumo" (Lambert, Stock e Ellram, 1998, p. 3).

A proposta de Pagh e Cooper (1998) para a análise da estratégia logística é uma exceção. Em seu artigo, o fluxo de produtos é decomposto nas dimensões tempo e espaço, nas quais podem ser tomadas decisões de antecipação e de postergação. Especificamente, decisões de centralização dos estoques (postergação) ou de descentralização (antecipação) podem ser tomadas na dimensão espaço. A dimensão espaço está associada à escolha da política de distribuição física dos produtos. Já na dimensão tempo, podem ser tomadas decisões de produzir contra-pedido (postergação) ou de produzir para estoque (antecipação). Em suma, a dimensão tempo está associada à escolha da política de produção.

Este artigo tem por objetivo testar e validar estatisticamente, em empresas brasileiras, a proposta conceitual de Pagh e Cooper (1998) para a estratégia logística e suas políticas principais. Por meio de entrevistas estruturadas com gerentes de logística de grandes empresas industriais foi possível coletar e compilar dados sobre as políticas de produção e de distribuição atualmente em curso, bem como sobre as principais características do produto, da operação e da demanda que influenciam essas políticas. Dentre os principais resultados obtidos está a quantificação e a hierarquização do impacto das principais características do negócio nas políticas de produção (para estoque/contra-pedido) e de distribuição (centralização/ descentralização). Esses resultados têm implicações acadêmicas e gerenciais relacionadas à confirmação estatística de diversas relações empíricas e ao direcionamento da atenção gerencial às características mais relevantes do negócio. Nas seções seguintes é apresentada uma descrição mais detal hada do referencial teórico, além dos objetivos e da metodologia utilizada na pesquisa. O utras duas seções são dedicadas à análise e à discussão dos resultados, sendo ao final apresentadas as conclusões e suas implicações acadêmicas e gerenciais.

\section{REFERENCIAL TEÓRICO}

\section{Política de distribuição física: centralização ou descentralização}

A revisão de literatura aponta algumas características do produto, da operação e da demanda que influenciam a decisão de centralização dos estoques. Dentre as características do produto, merece destaque a densidade de custos, que é a razão entre o custo do produto vendido e o peso ou volume do produto. De maneira geral, pode se afirmar que quanto maior a densidade de custos do produto, maior é a tendência para a centralização de seus estoques (Silver e Peterson, 1985, p. 384), ao passo que quanto menor a densidade de custos, maior é a tendência para a descentralização dos estoques (Ballou, 1992, p. 162; Christopher, 1997, p. 172). Por exemplo, quanto menor a densidade de custos, maior é a necessidade de minimizar os custos unitários de distribuição de modo a assegurar a competitividade do produto. A descentralização dos estoques viabiliza a consolidação de carregamentos e a conseqüente diluição dos custos fixos de distribuição por uma maior quantidade de produtos (Jayaraman, 1998).

Dentre as características da demanda que afetam a centralização dos estoques, destacam-se o giro dos estoques - razão entre as vendas anuais e o nível de estoque - e o coeficiente de variação das vendas - razão entre o desvio padrão e a média de vendas. Quanto maior o giro, maior a propensão para a descentralização dos estoques, basicamente por que menores são os riscos associados à obsolescência, perecibilidade ou encalhe de produtos. Por outro lado, quanto maior o coeficiente de variação das vendas, maior a propensão para a centralização dos estoques com o intuito de evitar, por exemplo, alocações equivocadas por diferentes instalações, ou seja, "enviar o produto errado para o local errado" (Silver e Peterson, 1985, p. 487; Zinn e Bowersox, 1989; Waters, 1992, p. 127; M entzer, Kahn e Bienstock, 1996, p. 36; Pagh e Cooper, 1998).

Ainda dentre as características da demanda, o tempo de entrega para o cliente pode influenciar a centralização dos estoques (Bowersox e Closs, 1996, p. 503). Por exemplo, supondo uma determinada disponibilidade de produto e um dado modal de transporte, tem- 
pos de entrega mais curtos e consistentes são al cançados por meio da descentralização dos estoques. Um curto tempo de entrega para o cliente também pode favorecer a descentralização dos estoques, na medida em que seja viável adiar a execução de determinadas atividades produtivas até a colocação do pedido pelo cliente e executá-las em outras instalações mais próximas deste. Em determinadas circunstâncias, as operações finais de mistura (tintas em lojas de materiais de construção), montagem (acessórios em veículos novos) e embalagem (alimentos e bebidas) podem ser postergadas até que haja clara definição a respeito de quais SKUs (stock keeping units - unidades de produtos em estoque) serão vendidas, eliminando-se, com isso, os riscos associados à incerteza da demanda futura.

\section{Política de produção: para estoque ou contra-pedido}

A política de produção envolve a escolha entre produzir para estoque ou produzir contra-pedido. Algumas características do produto, da operação e da demanda podem influenciar essa decisão, como, por exemplo, o grau de contato entre as operações e o cliente, o volume de produção, a duração do ciclo de vida do produto, o tempo de entrega exigido pel o cliente e o coeficiente de variação das vendas (Wemmerlov, 1984).

A exigência de curtos tempos de entrega leva à produção para estoque ( $\mathrm{i}$ i, 1992). Por outro lado, a possibilidade e o desejo de personalizar um produto levam à produção contra-pedido. Por meio de um alto grau de contato entre a área de produção e os clientes, os produtos acabados podem ser personalizados, de acordo com necessidades específicas, sendo produzidos contra-pedido a partir de uma configuração básica. Especificamente contra a personalização, existem dois obstáculos principais à produção contra-pedido, que favorecem a manutenção em estoque de um produto em sua configuração básica. Um deles é o coeficiente de variação das vendas, já que as vendas de um produto em sua configuração básica são mais previsíveis que as vendas de produtos personalizados (SimchiLevi e Kaminsky, 2000, p. 188). 0 outro é o custo adicionado por unidade: produtos em sua configuração básica implicam menor custo de manutenção de estoques que produtos personalizados.

Evidências empíricas apresentadas por Stalk (1988), Inman (1999), Cooper (1993) e Pagh e Cooper (1998) corroboram Wemmerlov (1984) ao apontar quatro fatores que deveriam ser observados para a produção contra-pedido. 0 primeiro del es é a razão en tre o tem- po de entrega do produto acabado para o cliente final e o tempo de entrega da matéria-prima mais crítica pelo fornecedor (razão entre prazos). Quanto maior essa razão, maior a propensão para a produção contra-pedido. Por exemplo, quanto maior a razão entre esses prazos, maior o tempo de entrega dos produtos para o cliente em comparação ao tempo de entrega das matérias-primas pelo fornecedor. Esses elementos favorecem a produção contra-pedido na medida em que há maior folga para a acomodação do tempo de resposta da produção no tempo de entrega para o cliente.

0 segundo fator é o custo do produto vendido (Lambert, Stock e Ellram, 1998, p. 124). Pode ser interpretado como o volume de capital de giro necessário para produzir uma unidade adicional. Quanto maior o custo do produto vendido, maior a propensão para produzir contra-pedido.

0 terceiro é o coeficiente de variação das vendas (Pagh e Cooper, 1998). Assim como na distribuição física, a maior variabilidade nas vendas pode contribuir para a postergação do fluxo de produtos na produção.

Finalmente, o quarto fator está relacionado à obsolescência e à perecibilidade dos estoques. Quanto maiores a obsolescência e a perecibilidade dos produtos acabados, maior a propensão para produzir contra-pedido, a fim de se evitarem perdas de estoque.

Além desses fatores, deve se destacar que a tecnologia de processo de produção pode afetar a decisão de produzir para estoque ou contra-pedido (Kwan, 1999; Zipkin, 2001). Basicamente porque os processos discretos - como o automotivo e el etroeletrônico - são mais flexíveis que os processos contínuos - como a siderurgia e petroquímica - com relação à postergação no tempo (Hayes e Wheelwright, 1984, p. 216; Landvater, 1997, p. 188).

\section{Proposta de Pagh e Cooper}

Pagh e Cooper (1998) apresentaram uma matriz 2x2 com quatro alternativas para a estratégia logística, obtidas a partir da combinação das possíveis escolhas nas políticas de produção e de distribuição física. Essa combinação pode refletir uma:

- política de antecipação total à demanda no espaço e no tempo (descentralizar/para estoque);

- política de postergação total à demanda no espaço e no tempo (centralizar/contra-pedido);

- política de antecipação no espaço e postergação no tempo (descentralizar/contra-pedido);

- política postergação no espaço e antecipação no tempo (centralizar/para estoque). 
Segundo os autores, cada uma dessas quatro políticas apresenta vantagens e desvantagens em termos de custos e de flexibilidade operacional. Por exemplo, a política "descentralizar/para estoque" é a mais adotada pelas empresas. Com base em previsões de venda, todas as operações de produção são realizadas antes do envio dos produtos aos centros de distribuição. Por sua vez, o envio é efetivado antes da colocação dos pedidos pelos clientes finais. Economias de escala na produção e na distribuição são a principal vantagem dessa política, uma vez que o processamento dos materiais/produtos ocorre sempre em grandes lotes. Por outro lado, o investimento em estoques é el evado, e são maiores os riscos de obsol escência e os gastos com transferências entre instalações. Produtos de baixa densidade de custos e de al to giro, como os bens de consumo não duráveis - alimentos, bebidas e produtos de higiene e limpeza - , constituem exemplos.

Por sua vez, a política "descentralizar/contra-pedido" equivale àquelas descritas por Bowersox e Closs (1996, p. 477) e por Zinn e Bowersox (1988). Os primeiros estágios da produção são centralizados, objetivando-se economias de escala. A descentralização é operacionalizada pelo envio dos estoques de produtos semi-acabados para diversos centros de distribuição próximos ao cliente final, e tem por objetivo garantir tempos de entrega reduzidos. A principal vantagem dessa política é a redução do número de SKUs e dos níveis de estoque de segurança de produtos acabados nos diversos centros de distribuição. No entanto, há um acréscimo nos custos dos estágios finais de produção em função da descentralização de operações, como, por exemplo, embalagem, montagem ou mistura. Há também aumento nos custos de processamento de pedidos em função da necessidade de coordenar a distribuição física com os estágios finais da produção. São exemplos dessa política a produção e a distribuição de tintas por lojas de material de construção.

Com relação à política "centralizar/para estoque", as operações de produção são centralizadas e direcionadas para a formação de estoques a partir de previsões de vendas, sendo executadas antes da distribuição física. As vantagens dessa política estão relacionadas com a redução dos níveis de estoque de produtos acabados em função da central ização e suas desvantagens com maiores custos de distribuição, em função da maior quantidade de envios, carregamentos fracionados e contratação de transporte expresso. As economias de escala na produção são preservadas. Essa política é comum em indústrias de processos contínuos, como a petroquímica, celulose e borracha.

E finalmente, na política "centralizar/contra-pedido", as operações de produção são totalmente centralizadas, e iniciadas contra-pedido. A principal vantagem é a redução dos níveis de estoque de matériasprimas, produtos semi-acabados e produtos acabados em todos os estágios produtivos. Economias de escala na produção são praticamente eliminadas, ainda que, em algumas circunstâncias, possam ser mantidas economias de escala na distribuição por meio da contratação de prestadores de serviço. Produtos de alto custo adicionado e de elevado coeficiente de variação das vendas constituem exemplos, assim como o caso Dell (Dell, 1999, p. 116).

Em linhas gerais, a proposta de Pagh e Cooper (1998) para a estratégia logística indica que, adotadas diferentes decisões de produção e distribuição, seguem-se os impactos esperados em termos de custos e flexibilidade operacional, e a conseqüente adequação a um determinado tipo de produto, operação ou setor.

\section{PROCEDIMENTOS METODOLÓGICOS}

Tomando como pontos de partida o referencial teórico e os objetivos de pesquisa apresentados anteriormente, foi estruturada e conduzida uma pesquisa de campo (F owler, 1993, p. 1) em grandes empresas brasileiras visando gerar estatísticas sobre diferentes características do negócio e decisões de estratégia logística. A população considerada para a pesquisa é a definida pelo conjunto das 500 empresas que compõem - Ranking Exame. A abrangência da pesquisa foi limitada aos setores industriais de bens de consumo duráveis e não duráveis que vendem necessariamente, mas não exclusivamente, para intermediários da cadeia (como atacadistas e varejistas). Dessa forma, foram desconsideradas as empresas dos setores primário e terciário da economia, além das empresas do setor secundário, não enquadradas nessa restrição.

As subpopulações (quantidade de empresas) englobadas na pesquisa de campo são as do setor químico e petroquímico (46), alimentício (40), automotivo (31), tecnologia e computação (26), el etroel etrônico (21) e farmacêutico (17). O tamanho total dessas subpopulações perfaz 181 empresas, ou 36,2\% do total de 500 empresas. Entretanto, se for considerado para efeito de definição populacional o conjunto de setores in- 
dustriais de bens de consumo duráveis e não duráveis que vendem necessariamente, mas não exclusivamente, para intermediários, o tamanho da população é de 254 empresas. As seis subpopulações consideradas na pesquisa perfazem $71,3 \%$ desse total.

Para cada setor foi selecionada uma amostra de conveniência de $10 \%$ das empresas listadas. Por exemplo, como no setor alimentício havia 40 empresas listadas, foram sel ecionadas as quatro primeiras empresas. Cada empresa selecionada foi convidada a participar do estudo. As empresas que recusaram foram substituídas pelas empresas seguintes na listagem de cada setor. A fração amostral final foi de $14,4 \%$ da população, sendo que as frações amostrais variaram entre um mínimo de $9,7 \%$ (automotivo) e um máximo $29,4 \%$ (farmacêutico), conforme indicado na Tabela 1.

Para medir o impacto das características do negócio nas políticas propostas por Pagh e Cooper (1998), foi conduzida uma série de entrevistas estruturadas com os gerentes de logística responsáveis pelas decisões de produção e de distribuição de cada empresa pesquisada. Cada gerente forneceu dados corporativos para dois SKUs e as entrevistas em cada empresa duraram cerca de uma hora e meia. Um dos SKUs é classificado como classe A em faturamento e outro como classe $C$. As entrevistas conduzidas foram estruturadas a partir de um questionário composto em sua totalidade por questões factuais. Questões de caráter factual simplificam consideravelmente o projeto do questionário, já que são eliminados aspectos como a formulação de diferentes alternativas para uma mesma pergunta. Cabe ressaltar que as questões factuais contidas no questionário reportam-se a eventos presentes, ou seja, características do negócio e decisões de produção e distribuição, que são familiares aos respondentes.

Os dados coletados estão resumidos na Tabela 2, onde são apresentadas estatísticas descritivas (médias e desvios padrão) para cada característica do negócio e para cada política considerada no questionário. $\mathrm{Na}$ Tabela 2 também é apresentada a operacionalização de cada uma dessas variáveis, o que inclui a criação de variáveis binárias ( 0 ou 1 ) para representar as decisões nas políticas propostas por Pagh e Cooper.

Técnicas de análise multivariada foram utilizadas para identificar o grupo ao qual pertence uma determinada variável, ou seja, para relacionar as características do negócio a uma determinada política ou decisão. Especificamente, a regressão logística é a técnica de análise multivariada mais apropriada quando a variável dependente (políticas) é nominal, categórica ou dummy, e as variáveis independentes (características do negócio) pertencem à escala de razão (Hair, Anderson e Tatham, 1998, p. 244). Essa técnica também é conhecida como análise logit, e é limitada em sua forma básica a apenas dois grupos. A regressão logística multinomial é capaz de lidar simultaneamente com mais de dois grupos. Ambas as análises de regressão logística, simples e multinomial, independem das premissas com relação à normalidade dos dados e igualdade das matrizes de covariância entre os grupos.

A análise dos resultados, detal hada na próxima seção, observou a seqüência descrita a seguir. Inicialmente a relação entre cada característica do negócio e cada política foi testada por análise de correlação. Seu objetivo foi identificar as relações estatisticamente significativas, bem como os sinais dessas relações. 0 segundo passo na análise dos resultados foi a regressão logística simples. Seus objetivos foram entender o efeito simultâneo das características do negócio sobre cada uma das políticas separadamente e ordenar as características do negócio por importância relativa. A regressão logística simples teve o objetivo adicional de validar o sinal e a significância dos resultados obtidos por

Tabela 1 - Tamanhos e frações amostrais coletados na pesquisa de campo

\begin{tabular}{|l|c|c|c|c|}
\hline \multicolumn{1}{|c|}{ SUBPOPULAÇÕES } & TAMANHOS & $\begin{array}{c}\text { TAMANHOS AMOSTRAIS } \\
\text { FINAIS }\end{array}$ & $\begin{array}{c}\text { FRAÇÕES AMOS TRAIS } \\
\text { FINAIS }\end{array}$ & $\begin{array}{c}\text { TOTAL DE SKUS } \\
\text { CLASSES A E C }\end{array}$ \\
\hline Químico e Petroquímico & 46 & 5 & $10,9 \%$ & 10 \\
\hline Alimentício & 40 & 5 & $12,5 \%$ & 10 \\
\hline Automotivo & 31 & 3 & $9,7 \%$ & 8 \\
\hline Eletroeletrônicos & 26 & 4 & $15,4 \%$ & 8 \\
\hline Tecnologia e Computação & 21 & 4 & $19,0 \%$ & 10 \\
\hline Farmacêutico & 17 & 5 & $29,4 \%$ & $\mathbf{5 2}$ \\
\hline Total & 181 & $\mathbf{2 6}$ & $\mathbf{1 4 , 4} \%$ & 8 \\
\hline
\end{tabular}


meio da análise de correlação, teste estatístico reconhecidamente mais fraco. Finalmente, o terceiro passo na análise dos resultados foi a regressão logística multinomial, tendo sido seu objetivo principal testar o impacto das características do negócio simultaneamente em todas as políticas propostas por Pagh e Cooper.
Deve se observar que todas as variáveis foram normalizadas para reduzir o efeito de multicolinearidade nas análises de regressão logística. Também deve se destacar que, em todos os testes estatísticos, o nível de significância adotado foi 0,10. Segundo Mentzer e Kahn (1995), esse nível de significância é geralmente aceito para pesquisas em logística.

Tabela 2 - Operacionalização das variáveis pesquisadas

\begin{tabular}{|c|c|c|c|c|c|}
\hline $\begin{array}{l}\text { CARACTERISTICAS/ } \\
\text { DECISÕES }\end{array}$ & $\begin{array}{l}\text { VARIÁVEIS COLETADAS } \\
\text { INICIALMENTE }\end{array}$ & $\begin{array}{c}\text { VARIÁVEIS } \\
\text { TRANSFORMADAS }\end{array}$ & FÓRMULA & MÉDIA & $\begin{array}{l}\text { DESVIO } \\
\text { PADRÃOO }\end{array}$ \\
\hline \multirow[t]{4}{*}{$\begin{array}{l}\text { Características } \\
\text { do Produto }\end{array}$} & $\begin{array}{l}\text { Custo do Produto Vendido - } \\
\text { CPV (\$) }\end{array}$ & \multirow[t]{2}{*}{$\begin{array}{l}\text { Densidade de } \\
\text { Custos }\end{array}$} & \multirow[t]{2}{*}{$\begin{array}{l}=\mathrm{CPV} / \text { (Peso ou } \\
\text { Volume) }\end{array}$} & \multirow[t]{2}{*}{$21.458,26$} & \multirow[t]{2}{*}{$77.435,09$} \\
\hline & $\begin{array}{l}\text { Peso }(\mathrm{kg}) \text { ou Volume do Pro- } \\
\text { duto }\left(\mathrm{m}^{3}\right)\end{array}$ & & & & \\
\hline & $\begin{array}{l}\text { Duração do Ciclo de Vida } \\
\text { (Meses) }\end{array}$ & $\begin{array}{l}\text { Grau de } \\
\text { Obsolescência }\end{array}$ & $\begin{array}{l}=1 / \text { (Duração } \\
\text { do Ciclo de Vida) }\end{array}$ & 0,03 & 0,05 \\
\hline & Prazo de Validade (Meses) & $\begin{array}{l}\text { Grau de } \\
\text { Perecibilidade }\end{array}$ & $\begin{array}{l}=1 / \text { (Prazo de } \\
\text { Validade) }\end{array}$ & 0,14 & 0,29 \\
\hline \multirow[t]{4}{*}{$\begin{array}{l}\text { Características } \\
\text { da Demanda }\end{array}$} & $\begin{array}{l}\text { Venda Mensal Máxima } \\
\text { (unidades) }\end{array}$ & \multirow{3}{*}{$\begin{array}{l}\text { Coeficiente de } \\
\text { variação das } \\
\text { Vendas }\end{array}$} & \multirow{2}{*}{$\begin{array}{l}=\text { (Desvio Padrão das } \\
\text { Vendas) / (Média } \\
\text { das Vendas) }\end{array}$} & \multirow[t]{2}{*}{0,4499} & \multirow[t]{2}{*}{0,176} \\
\hline & $\begin{array}{l}\text { Venda Mensal Mínima } \\
\text { (unidades) }\end{array}$ & & & & \\
\hline & \multicolumn{2}{|l|}{ Giro (vezes/ ano) } & & 29,00 & 33,16 \\
\hline & $\begin{array}{l}\text { Tempo de Entrega para Prin- } \\
\text { cipal Cliente a partir da Co- } \\
\text { locação do Pedido (Dias) }\end{array}$ & $\begin{array}{l}\text { Razão entre } \\
\text { Prazos }\end{array}$ & $\begin{array}{l}\text { = Tempo de Entrega } \\
\text { para Principal Clien- } \\
\text { te } / \text { Tempo de Entre- } \\
\text { ga do Insumo Mais } \\
\text { Demorado }\end{array}$ & 0,16 & 0,33 \\
\hline \multirow[t]{2}{*}{$\begin{array}{l}\text { Características } \\
\text { da Operação }\end{array}$} & $\begin{array}{l}\text { Tempo de Entrega do Insumo } \\
\text { Mais Demorado a partir da } \\
\text { Colocação do Pedido (Dias) }\end{array}$ & & & & \\
\hline & \multicolumn{3}{|c|}{$\begin{array}{l}\text { Tecnologia de processo } \\
\text { Se Contínuo = } 1 \\
\text { (Alimentício, Farmacêutico, e Químico e Petroquímico) } \\
\text { Se Discreto = } 0 \\
\text { (Automotivo, Eletroeletrônico, e Tecnologia e Computação) }\end{array}$} & 0,58 & 0,25 \\
\hline \multirow[t]{2}{*}{$\begin{array}{l}\text { Política de } \\
\text { Distribuição }\end{array}$} & \multirow{2}{*}{$\begin{array}{l}\text { Se Descentralizar = } 1 \\
\text { (antecipação no espaço) } \\
\text { Se Centralizar = } 0 \\
\text { (postergação no espaço) }\end{array}$} & \multicolumn{2}{|c|}{ Descentralizar/ Para estoque } & 0,48 & 0,51 \\
\hline & & \multicolumn{2}{|c|}{ Descentralizar/ Contra-pedido } & 0,14 & 0,36 \\
\hline \multirow[t]{2}{*}{$\begin{array}{l}\text { Política de } \\
\text { Produção }\end{array}$} & \multirow{2}{*}{$\begin{array}{l}\text { Se Contra-pedido =0 } \\
\text { (postergação no tempo) } \\
\text { Se Para estoque = } 1 \\
\text { (antecipação no tempo) }\end{array}$} & \multicolumn{2}{|c|}{ Centralizar/ Para estoque } & 0,29 & 0,46 \\
\hline & & \multicolumn{2}{|c|}{ Centralizar/ Contra-pedido } & 0,09 & 0,29 \\
\hline
\end{tabular}




\section{ANÁLISE DOS RESULTADOS}

\section{Análises de correlação}

Foram conduzidas análises de correlação entre as quatro políticas propostas por Pagh e Cooper (1998) e as características do negócio para os 52 SKUs pesquisados. A política "descentralizar/para estoque" é estatisticamente correlacionada com o coeficiente de variação das vendas $(-0,395 * ; 48)$, 0 tempo de entrega $(-0,337 * *$; $52)$, a razão entre prazos $(-0,302 * * ; 46)$ e o custo do produto vendido $(-0,256 ; 43)$. 0 primeiro valor entre parênteses corresponde ao coeficiente de correl ação e 0 segundo, ao tamanho da amostra considerado. Dois asteriscos representam significância de 0,05 e um asterisco representa significância de 0,01 . Nenhum asterisco indica significância de 0,10. 0 sinal dessas correlações indica que a adoção da política "descentral izar/para estoque" está relacionada a produtos com baixo coeficiente de variação, curto tempo de entrega, pequena razão entre prazos e baixo custo do produto vendido.

A política "centralizar/para estoque" é estatisticamente correlacionada com o giro $(-0,268 ; 46)$ e a tecnologia de processos $(0,234 ; 52)$. 0 sinal dessas correlações indica que a adoção da política "centralizar/ para estoque" está relacionada a produtos de baixo giro, fabricados por processos contínuos.

A política "descentralizar/contra-pedido" é estatisticamente correlacionada com o tempo de entrega $(0,441 *$; $52)$, o coeficiente de variação das vendas $(0,341 * * ; 48)$, a tecnologia de processos $(-0,282 * * ; 52)$ e o giro $(0,263$; 46). 0 sinal dessas correlações indica que a adoção da política "descentralizar/contra-pedido" está relacionada a produtos com longo tempo de entrega, al to coeficiente de variação das vendas, fabricados por processos discretos e com elevado giro dos estoques.

Finalmente, a política "centralizar/contra-pedido" é estatisticamente correlacionada com o grau de obsolescência $(0,383 * ; 48)$, a tecnologia de processos $(-0,337 * * ; 52)$, a razão entre prazos $(0,343 * * ; 46)$ e 0 tempo de entrega $\left(0,315^{* *} ; 52\right)$. 0 sinal dessas correlações indica que a adoção da política "centralizar/ contra-pedido" está relacionada a produtos com al to grau de obsolescência, fabricados por processos discretos, com el evada razão entre prazos e longo tempo de entrega.

As análises de correlação sugerem que diferentes combinações de características do negócio levariam à adoção de uma determinada política. Em outras palavras, parece que a adoção dessas políticas não está associada a um conjunto homogêneo de características.

\section{Análises de regressão logística simples}

Foram conduzidas regressões logísticas simples para determinar o efeito simultâneo das características do negócio na escolha de uma determinada política quando as demais são agrupadas em uma única alternativa. Em outras palavras, buscou-se determinar as características do negócio que favoreceriam ou não a adoção de uma política específica.

De acordo com os resultados da Tabela 3, a política "descentralizar/para estoque" é significativamente correlacionada $(p<0,10)$ com 0 tempo de entrega e 0 coeficiente de variação das vendas. Os sinais negativos do tempo de entrega e do coeficiente de variação das vendas indicam que a política "descentralizar/para estoque" tende a ser adotada quando o tempo de entrega é curto e o coeficiente de variação das vendas é pequeno. De acordo com o $\mathrm{R}^{2}$ de Nagelkerke, essas duas variáveis explicam $50,6 \%$ da escolha dessa política.

As empresas evitam a política "descentralizar/para estoque" quando o coeficiente de variação das vendas é maior que 0,70 , independentemente do tempo de entrega; e quando o tempo de entrega é superior a 2,75 dias, independentemente do coeficiente de variação das vendas. Esses valores são determinados, respectivamente, pela interseção da linha que representa o ponto de corte $(p=0,50)$ com os eixos vertical e horizontal. A região com maior probabilidade para a adoção

Tabela 3 - Resultados da regressão logística: política descentralizar/ para estoque

\begin{tabular}{|l|l|c|c|c|}
\hline \multicolumn{1}{|c|}{ DECISÃOO } & \multicolumn{1}{|c|}{ VARIÁVEL } & WALD & SIGNIFICÂNCIA \\
\hline $1=\operatorname{sim}$ & Constante & $-3,935$ & 5,761 & 0,016 \\
\hline 0 não & Tempo de Entrega & $-11,853$ & 6,333 & 0,012 \\
\hline & Coeficiente de Variação das Vendas & $-0,734$ & 2,707 & 0,098 \\
\hline
\end{tabular}

Variável dependente: descentralizar/ para estoque

Cox \& Snell $R^{2}=0,378$

Nagelkerke $R^{2}=0,506$

Qui-quadrado para 0 modelo $=22,814($ Sig. $=0,000)$ 
dessa política $(p>0,95)$ é delimitada pela relação do tempo de entrega com o coeficiente de variação das vendas, com um tempo de entrega máximo de 0,70 dia e um coeficiente de variação das vendas máximo de 0,18 . A Tabela 3 é esclarecedora com relação à quantificação das oportunidades para se explorarem economias de escala simultaneamente na produção e na distribuição (antecipação total): um ambiente com relativamente pouca oscilação nas vendas e com curto tempo de entrega. 0 exemplo paradigmático dessa política seria a cadeia de produção e de distribuição de combustíveis.

Com base nos resultados da Tabela 4, a política "central izar/para estoque" é significativamente correlacionada com a tecnologia de processos e o giro dos estoques $(p<0,10)$. 0 sinal negativo do giro dos estoques e o sinal positivo da tecnologia de processos indicam que essa política tende a ser adotada quando o processo de produção é contínuo e o giro dos estoques de produto acabado é pequeno. De acordo com o $\mathrm{R}^{2}$ de $\mathrm{N}$ agel kerke, essas variáveis explicam $21,6 \%$ das escoIhas dessa política.

De acordo com os resultados da Tabela 5, a política "descentralizar/contra-pedido" é significativamente correlacionada com o tempo de entrega, o coeficiente de variação das vendas e a interação de primeira ordem entre essas duas variáveis. 0 sinal positivo do tempo de entrega e do coeficiente de variação das vendas indica que um longo tempo de entrega e um al to coeficiente de variação das vendas diferencia essa política das demais. Entretanto, a interação aponta que quanto maiores essas variáveis, menor a tendência para a adoção dessa política. Isso indica que há um limite para a sua adoção quando o tempo de entrega é extremamente longo e o coeficiente de variação das vendas é extremamente al to.

Ainda de acordo com os resultados da Tabela 5, as empresas tendem a adotar essa política quando o coeficiente de variação das vendas é alto $(>1,25)$ e o tempo de entrega é inferior a 10 dias, sendo quantificadas as oportunidade para a descentral ização dos estoques e de operações finais de produção pouco intensivas em capital: montagem, embalagem, mistura e pintura. Os exemplos paradigmáticos dessa política são os casos da Hewlett-Packard (embalagem final dos manuais) e da mistura final dos pigmentos às bases na fabricação das tintas em varejistas de materiais de construção.

Por sua vez, de acordo com os resultados da Tabela 6, a política "centralizar/contra-pedido" é significativamente correlacionada com o grau de obsolescência

Tabela 4 - Resultados da regressão logística: política centralizar/ para estoque

\begin{tabular}{|l|l|c|c|c|}
\hline \multicolumn{1}{|c|}{ DECISÃO } & \multicolumn{1}{|c|}{ VARIÁVEL } & B & WALD & SIGNIFICÂNCIA \\
\hline $1=\operatorname{sim}$ & Constante & $-1,667$ & 6,129 & 0,013 \\
\hline$=$ não & Giro dos Estoques & $-1,301$ & 3,212 & 0,073 \\
\hline & Tecnologia de Processos & 1,248 & 2,865 & 0,091 \\
\hline
\end{tabular}

Variável dependente: centralizar/ para estoque

Cox \& Snell $R^{2}=0,157$

Nagelkerke $R^{2}=0,216$

Qui-quadrado para o modelo $=7,845($ Sig. $=0,020)$

Tabela 5 - Resultados da regressão logística: política descentralizar/ contra-pedido

\begin{tabular}{|l|l|r|r|r|}
\hline \multicolumn{1}{|c|}{ DECISÃO } & \multicolumn{1}{|c|}{ VARIÁVEL } & B & WALD & SIGNIFICÂNCIA \\
\hline $1=\operatorname{sim}$ & Constante & $-1,867$ & 18,510 & 0,000 \\
\hline 0 não & Tempo de Entrega & 3,705 & 5,879 & 0,015 \\
\hline & Coeficiente de Variação das Vendas & 0,988 & 3,133 & 0,077 \\
\hline & Tempo de Entrega*Coeficiente de & $-1,870$ & 6,111 & 0,013 \\
\hline & Variação das Vendas & & \\
\hline
\end{tabular}

Variável dependente: descentralizar/ contra-pedido

Cox \& Snell $R^{2}=0,249$

Nagelkerke $R^{2}=0,470$

Qui-quadrado para o modelo $=13,729($ Sig. $=0,003)$ 
$(p<0,05)$. 0 sinal positivo da variável explicativa indica que um el evado grau de obsolescência (ciclo de vida de curta duração) é a única característica do negócio que diferencia significativamente essa política das demais.

As empresas tendem a adotar essa política quando a duração do ciclo de vida do produto é menor que 8,5 meses (quando se controla essa variável pelo tempo de entrega e pelo coeficiente de variação das vendas) e menor que 4,5 meses (sem esse controle). Em outras palavras, as oportunidades para a adoção dessa política estariam concentradas em situações em que 0 grau de obsolescência do produto é consideravel mente al to. 0 exemplo paradigmático dessa política seria a Dell Computers.

\section{Análises de regressão logística multinomial}

U ma análise com a política "descentralizar/para estoque" como a categoria de referência foi conduzida para determinar as características do produto, da operação e da demanda simultaneamente relacionadas com a adoção de todas as políticas. Os resultados são apresentados na Tabela 7.
De acordo com essa tabela, todas as políticas são estatisticamente correlacionadas com o tempo de entrega $(p<0,05)$, quando se considera a política "descentralizar/para estoque" como a categoria de referência. Além disso, o tempo de entrega é a única característica do negócio que explica simultaneamente todas as políticas analisadas. Esse resultado reflete, de certo modo, a natureza heterogênea dos conjuntos de características do negócio estatisticamente correlacionados com cada política, conforme foi apontado pelas análises de correlação e de regressão logística simples.

Como os coeficientes do tempo de entrega para as três políticas são positivos, um maior tempo de entrega parece favorecer a adoção dessas três outras políticas comparativamente à política "descentralizar/para estoque". Em outras palavras, a política "descentralizar/para estoque" está posicionada no início da escala do tempo de entrega, conforme proposição de Pagh e Cooper (1998) e Bowersox e Closs (1996, p. 477). Por outro lado, a política "centralizar/contra-pedido" apresenta o maior coeficiente de tempo de entrega. Isso indica, de acordo com esses autores, que essa política

Tabela 6 - Resultados da regressão logística: política centralizar/ contra-pedido

\begin{tabular}{|l|l|r|r|c|}
\multicolumn{1}{|c|}{ DECISÃO } & \multicolumn{1}{|c|}{ VARIÁVEL } & B & WALD & SIGNIFICÂNCIA \\
\hline $1=\operatorname{sim}$ & Constante & $-2,730$ & 18,233 & 0,000 \\
\hline 0 não & Grau de Obsolescência & 0,847 & 4,268 & 0,039 \\
\hline
\end{tabular}

Variável dependente: Centralizar/ Contra-pedido

Cox \& Snell $R^{2}=0,091$

Nagelkerke $R^{2}=0,209$

Qui-quadrado para o modelo $=4,593($ Sig. $=0,032)$

Tabela 7 - Resultados da regressão logística multinomial para quatro políticas

\begin{tabular}{|l|l|l|l|l|}
\hline \multicolumn{1}{|c|}{ POLITICA } & VARIÁVEL & B & WALD & SIGNIFICÂNCIA \\
\hline Centralizar/ Contra-pedido & Constante & 1,185 & 0,720 & 0,396 \\
\hline & Tempo de Entrega & 12,420 & 9,612 & 0,002 \\
\hline \multirow{2}{*}{ Centralizar/ Para estoque } & Constante & 2,490 & 3,817 & 0,051 \\
\hline & Tempo de Entrega & 9,042 & 5,849 & 0,016 \\
\hline \multirow{2}{*}{ Descentralizar/ Contra-pedido } & Constante & 1,900 & 2,038 & 0,153 \\
\hline & Tempo de Entrega & 12,400 & 9,609 & 0,002 \\
\hline
\end{tabular}

Categoria de referência: Descentralizar/ Para estoque

Cox \& Snell $R^{2}=0,452$

Nagelkerke $R^{2}=0,496$

Qui-quadrado para o modelo $=31,245($ Sig. $=0,000)$

Graus de liberdade $=3$ 
está posicionada ao final da escala do tempo de entrega. Dessa forma, as políticas "centralizar/para estoque" e "descentralizar/contra-pedido" estão posicionadas entre os extremos dessa escala de tempos de entrega. As curvas de probabilidade para a escolha de cada política são apresentadas na Figura 1, construído a partir da Tabela 7. O ponto de corte para a política "centralizar/para estoque" é 3,18 dias; para "descentralizar/contra-pedido", 4,36 dias; e para "central izar/contra-pedido", 4,98 dias.

Ainda de acordo com os resultados, um número excessivo de políticas pode estar diluindo o poder de explicação de outras variáveis que não o tempo de entrega. Dessa forma, outra regressão logística multinomial foi conduzida agrupando-se as políticas "centralizar/para estoque" e "descentralizar/contrapedido" na categoria de referência. Seu objetivo é quantificar o papel simultâneo do tempo de entrega, do coeficiente de variação das vendas e do grau de obsolescência na escol ha das políticas "descentral izar/ para estoque" e "centralizar/contra-pedido", ou seja, antecipação total e postergação total. O s resultados são apresentados na Tabela 8.

Os resultados da Tabela 8 indicam que o grau de obsolescência é a única característica do negócio que explica simultaneamente a escol ha das políticas "descentralizar/para estoque" e "centralizar/contra-pedi- do". Um maior grau de obsolescência parece estar associado à política "centralizar/contra-pedido", observando seu controle pelo tempo de entrega e pelo coeficiente de variação das vendas. 0 tempo de entrega e o coeficiente de variação das vendas são estatisticamente significativos para a escolha da política "descentralizar/para estoque". Produtos com curto tempo de entrega, pequeno coeficiente de variação das vendas e menor grau de obsolescência parecem estar associados à política de antecipação total.

Na Figura 2, construído a partir da Tabela 8, é apresentada a adoção das políticas "descentralizar/para estoque" e "centralizar/contra-pedido". As linhas de indiferença $(p=0,50)$ ilustram o efeito simultâneo dessas três características do negócio na escolha da política "descentralizar/para estoque" e o controle do grau de obsolescência pelo tempo de entrega e pelo coeficiente de variação das vendas na escolha da política "centralizar/contra-pedido". O ponto de corte na duração do ciclo de vida para a escolha da política "centralizar/contra-pedido" é 8,16 meses. Quando a duração do ciclo de vida do produto é de, por exemplo, 24 meses, a linha tracejada indica diferentes combinações do coeficiente de variação das vendas e do tempo de entrega para $p=0,50$, ou seja, para o ponto de corte na escolha da política "descentralizar/para estoque".

Figura 1 - Curvas de probabilidade para adoção das políticas em função do tempo de entrega

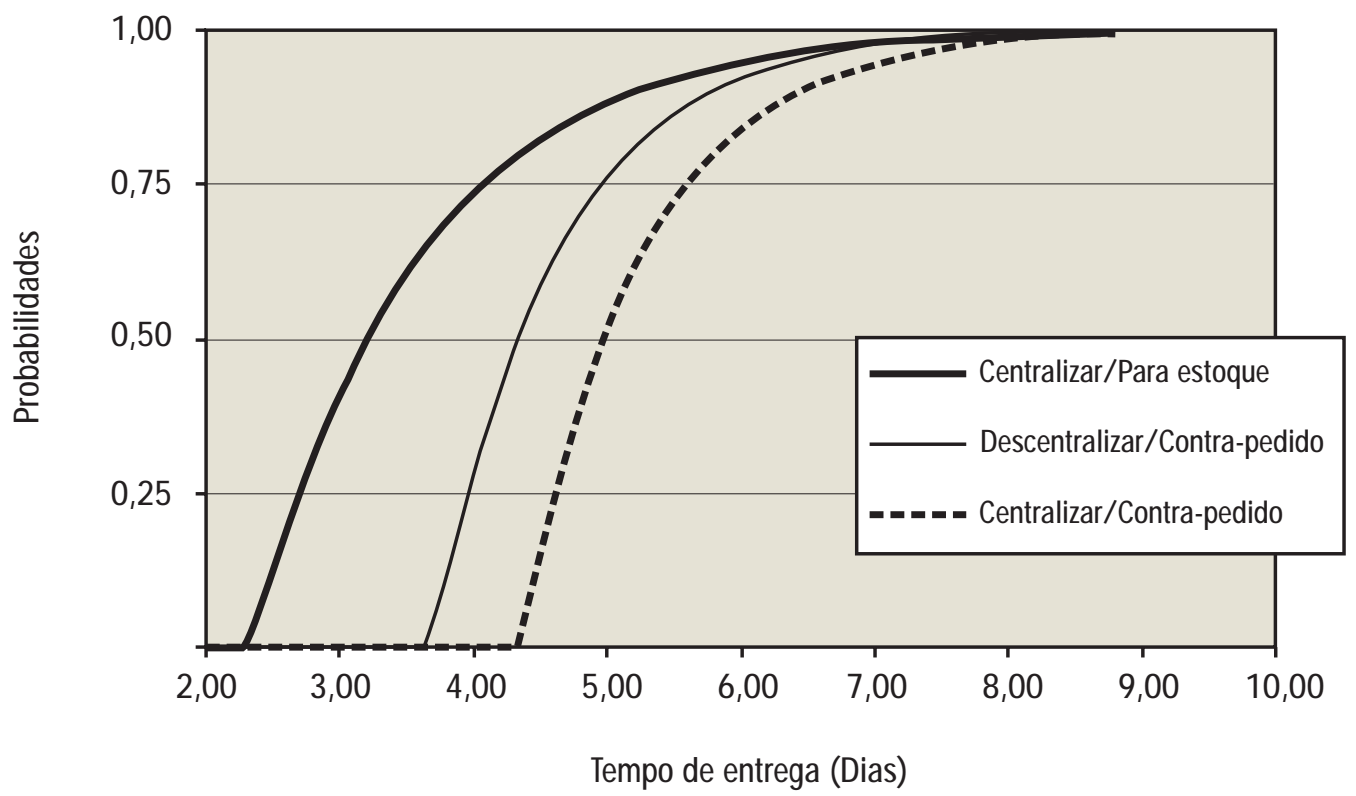




\section{DISCUSSÃO}

De acordo com Ghemawat (1991, p. 103), a avaliação da sustentabilidade de uma estratégia é um exercício lógico eminentemente estático, no qual são construídos diferentes cenários a partir do comportamento futuro das variáveis mais importantes para se compreender 0 negócio. Nesse sentido, os resultados apresentados permitem inferir a sustentabilidade de uma determinada política, ou seja, sua adequação para diferentes valores do tempo de entrega, do coeficiente de variação das vendas e do grau de obsolescência. Esses resultados permitem responder, por exemplo, à seguinte questão: se os tempos de entrega forem aumentados ou reduzidos substancialmente, a política em curso continua sendo adequada? O utra questão que pode ser

Tabela 8 - Resultados da regressão logística multinomial para três políticas

\begin{tabular}{|l|l|r|r|c|}
\hline \multirow{2}{*}{ POLÍTCA } & \multicolumn{1}{|c|}{ VARIÁVEL } & W & WALD & \multicolumn{1}{c|}{ SIGNIFICÂNCIA } \\
\hline \multirow{5}{*}{ Cescentralizar/ Para estoque } & Constante & $-3,345$ & 5,356 & 0,021 \\
\hline & Tempo de Entrega & $-11,002$ & 6,394 & 0,011 \\
\hline & Grau de Obsolescência & 1,307 & 3,174 & 0,075 \\
\hline & Coeficiente de Variação das Vendas & $-0,947$ & 2,621 & 0,100 \\
\hline & Constante & $-2,315$ & 7,036 & 0,008 \\
\hline & Tempo de Entrega & 0,549 & 0,391 & 0,532 \\
\hline & Grau de Obsolescência & 1,632 & 3,775 & 0,052 \\
\hline & Coeficiente de Variação das Vendas & $-1,039$ & 0,933 & 0,334 \\
\hline
\end{tabular}

Categoria de referência: Políticas sem ênfase definida na postergação e na antecipação

Cox\& Snell $R^{2}=0,497$

Nagelkerke $R^{2}=0,588$

Qui-quadrado para o modelo $=30,223($ Sig. $=0,000)$

Graus de liberdade $=6$

Figura 2 - Linhas de indiferença para as políticas em função do tempo de entrega, do coeficiente de variação das vendas e do ciclo de vida

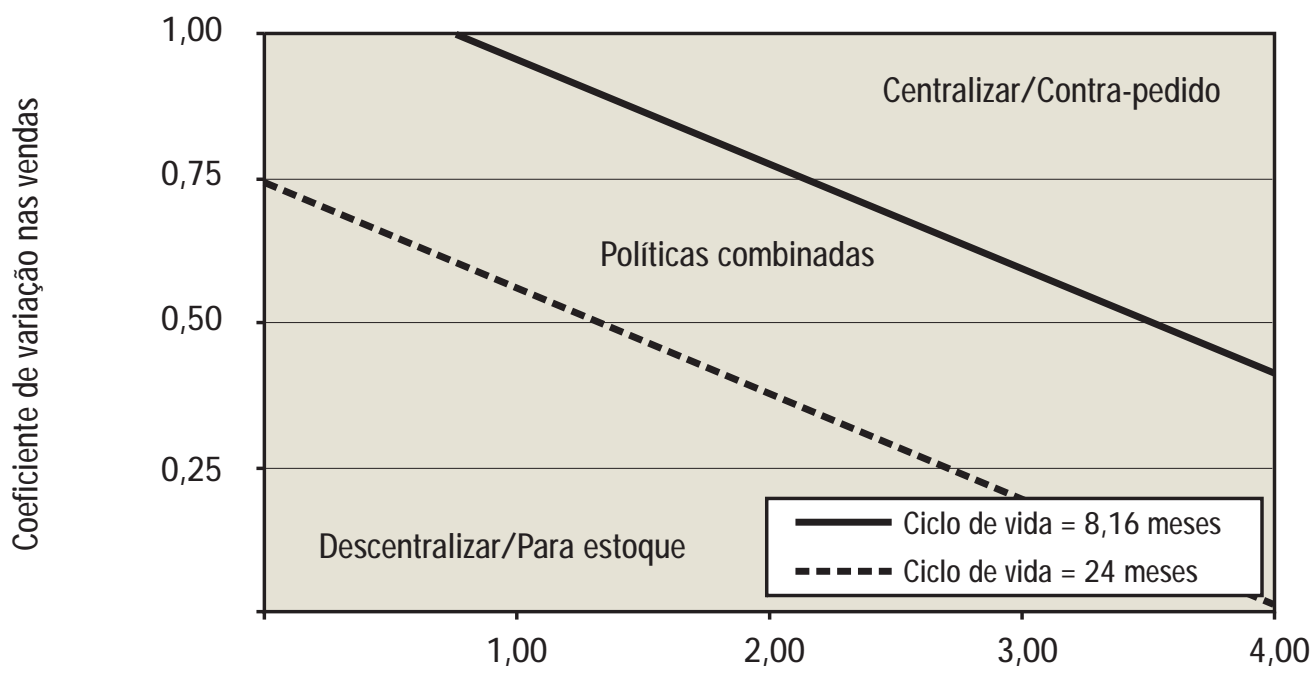

Tempo de entrega (Dias) 
respondida é: quais as chances de adoção de uma determinada política, dado um conjunto de características do negócio?

A avaliação da sustentabilidade também pode fornecer indícios da flexibilidade para mudar o curso de determinada política ou decisão. Por exemplo, a decisão de distribuição física (centralização/descentralização) parece ter maior flexibilidade de mudança que a decisão de produção (para estoque/contrapedido), quando a variável analisada é o tempo de entrega (veja a Figura 1). Isso porque, ao se observar na figura a seqüência "descentralizar/para estoque", "centralizar/para estoque", "descentralizar/contra-pedido" e "centralizar/contra-pedido", verificada com 0 aumento do tempo de entrega, a decisão de distribuição é alterada duas vezes, enquanto a decisão de produção, apenas uma vez. Essa maior flexibilidade pode ser conseqüência, por exemplo, de uma menor complexidade das operações de distribuição comparativamente às de produção. A reconhecida facilidade para terceirização e contratação de prestadores de serviço na distribuição poderia ser um indício de sua menor complexidade.

Os resultados obtidos também permitem evidenciar e quantificar o papel determinante do tempo de entrega na escol ha das políticas anal isadas. Em segundo plano, permitem destacar o papel do coeficiente de variação das vendas e do grau de obsolescência nessa escolha. Em resumo, de todas as características do negócio apresentadas no referencial teórico, apenas essas três variáveis são efetivamente responsáveis por explicar a maior parcela da variação na escolha dessas políticas (aproximadamente $60 \%$, conforme valor do $\mathrm{R}^{2}$ indicado na Tabela 8).

$M$ ais especificamente, esses resultados destacam-se de pesquisas anteriores rel atadas na literatura pela: (1) quantificação de relações entre as características do negócio e as políticas anal isadas; (2) validação dos sinais dessas relações; (3) determinação de modelos probabilísticos; e (4) mensuração em empresas brasileiras.

Além desses avanços acadêmicos, os resultados obtidos também constituem avanços gerenciais, uma vez que permitem: (1) direcionar a atenção dos gerentes às características mais relevantes para a tomada de decisão; (2) avaliar a adequação de iniciativas gerenciais, refletidas nas decisões de produção e distribuição física, para diferentes valores dessas características; e (3) segmentar as políticas por diferentes produtos e mercados.
Por exemplo, a partir dos resultados da Tabela 8, é possível inferir que um produto com coeficiente de variação das vendas de 0,5, tempo de entrega de 2 dias e ciclo de vida de 24 meses possui $48,85 \%$ de probabilidade (P1) de observar a política "descentralizar/para estoque" e 8,37\% de probabilidade (P2) de "centralizar/contra-pedido". Ambas as probabilidades são relativas à categoria de referência, conforme indicado nos cálculos a seguir para os valores normalizados dessas três variáveis. Os coeficientes da Tabela 8 estão destacados.

Descentralizar/ para estoque

$P 1=\exp (-\mathbf{3 , 3 4 5}-\mathbf{1 1 , 0 0 2} *((2-5,34) / 11,26)+$

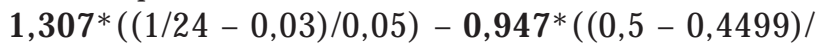
$0,176)) /(1+\exp (-\mathbf{3 , 3 4 5}-\mathbf{1 1}, \mathbf{0 0 2} *((2-5,34) / 11,26)$ $+1,307 *((1 / 24-0,03) / 0,05)-0,947 *((0,5-0,4499) /$ $0,176)))$

$\mathrm{P} 1=48,85 \%$

Centralizar/ contra-pedido

$\mathrm{P} 2=\exp (\mathbf{- 2 , 3 1 5}+\mathbf{0 , 5 4 9} *((2-5,34) / \mathbf{1 1}, 26)+$ $1,632 *((1 / 24-0,03) / 0,05)-1,039 *((0,5-0,4499) /$ $0,176)) /(1+\exp (-\mathbf{2 , 3 1 5}+\mathbf{0 , 5 4 9} *((2-5,34) / 11,26)$ $+1,632 *((1 / 24-0,03) / 0,05)-1,039 *((0,5-0,4499) /$ $0,176)))$

$\mathrm{P} 2=8,37 \%$

Dessa forma, percebe-se que, a partir dos resultados gerados, os gerentes têm subsídios para a tomada de decisões estratégicas em logística. As figuras apresentadas podem, por exemplo, ser utilizadas como instrumentos de apoio à escol ha das políticas analisadas. Esses resultados, entretanto, devem ser considerados como o ponto de partida do processo decisório, já que não refletem necessariamente as melhores práticas, apenas as práticas de mercado da amostra analisada.

\section{CONSIDERAÇÕES FINAIS}

A partir da pesquisa de campo, foi possível estabelecer modelos quantitativos para duas perspectivas de análise relacionadas com a escolha das políticas propostas por Pagh e Cooper (1998). A primeira perspectiva diz respeito à escolha de uma política quando as demais são agrupadas numa outra única política, sendo desenvolvida por meio de análise de regressão logística simples. Com base nessa análise foi possível determinar as linhas de indiferença $(p=0,50)$ e de 
probabilidade 0,95, para adoção de uma determinada política. Especificamente, foram quantificadas as relações entre o tempo de entrega e o coeficiente de variação das vendas para as políticas "descentralizar/para estoque" e "descentralizar/contra-pedido"; o grau de obsolescência para a política "centralizar/contra-pedido"; e o giro dos estoques e a tecnologia de processos para a política "centralizar/para estoque". Além disso, também foi possível quantificar as oportunidades para adotar as iniciativas verificadas na Dell e na HewlettPackard, bem como para adotar políticas "mais tradicionais" ("descentralizar/para estoque" e "centralizar/ para estoque").

A segunda perspectiva diz respeito à análise simultânea, por regressão logística multinomial, de todas as políticas. Com base nessa análise foi possível determinar a adequação de uma política em comparação com as demais, para diferentes valores das características do negócio. Merece destaque o modelo para as quatro políticas e o tempo de entrega apresentado na Tabela 7. Também foi possível corroborar o entendimento de Pagh e Cooper (1998) e Bowersox e Closs (1996, p. 477) sobre a adequação de cada política no continuum definido pelo tempo de entrega, inferindo, dessa forma, que a decisão de distribuição (centralizar/descentralizar) possui maior flexibilidade à mudança que a decisão de produção (para estoque/contra-pedido), quando se considera o tempo de entrega como base para a tomada de decisão.

As implicações dos resultados da pesquisa de campo estão relacionadas não apenas com as hipóteses que podem ser testadas por meio de estudos futuros, mas também com as novas linhas de pesquisa, derivadas da pesquisa atual. U ma linha de pesquisa futura, por exemplo, estaria relacionada com a comparação de como a relação entre as características do negócio e as políticas analisadas pode variar entre as práticas de mercado e as melhores práticas, ou entre o Brasil e outros países. Ainda que os sinais dessas relações, determinados nos model os quantitativos, corroborem as evidências empíricas descritas na literatura, é possível que seus coeficientes sejam significativamente diferentes quando forem comparados com as práticas de mercado para as melhores práticas, ou comparando-se o Brasil com outros países. Essa diferença entre os coeficientes indicaria possíveis diferenças no peso ou impacto de cada uma das características do negócio na tomada de decisão, mesmo que seu sentido (sinal) seja idêntico.

A principal limitação dos resultados da pesquisa de campo é a representatividade da amostra. Conforme apontado na discussão dos resultados, os modelos representam as práticas de mercado dos seis setores pesquisados no Brasil, no sentido de como as características do negócio afetariam, em média, as políticas propostas por Pagh e Cooper, não sendo possível a identificação das melhores ou das piores práticas ou mesmo a comparação com resultados de outros países. A representatividade, portanto, demarca as fronteiras da validação externa dos resultados e do potencial gerencial para a utilização dos modelos.

\section{REFERÊNCIAS BIBLIOGRÁFICAS}

ABAD, P. L. O ptimal pricing and lot-sizing under conditions of perishability, finite production and partial backordering and lost sale. European Journal of Operations Research, v. 144, n. 3, p. 677-686, 2003.

BALLOU, R. H. Business Logistics M anagement. New York: Prentice Hall, 1992.

BOWERSOX, D. J.; CLOSS, D. J. Logistical Management: The Integrated Supply Chain Process. New York: McGraw-Hill, 1996.

CHRISTOPHER, M. Logística e gerenciamento da cadeia de suprimentos: estratégias para redução de custos e melhoria dos serviços. São Paulo: Pioneira, 1997.

COCHRAN, W. G. Sampling Techniques. New York: Wiley \& Sons, 1963.

COOPER, J. Logistics strategies for global business. International Journal of Physical Distribution and Logistics M anagement, v. 23, n. 4, p. 12-23, 1993.

DELL, M. Direct from D ell. New York: Harperbusiness, 1999.

FOW LER, F. J. Survey Research M ethods. Thousand Oaks, CA: Sage, 1993.

GHEM AWAT, P. Commitment: The Dynamic of Strategy. New York: Free Press, 1991.

HAIR, J. F.; ANDERSON, R. E.; TATHAM, R. L. Multivariate Data Analysis. New York: Prentice Hall, 1998.

HAYES, R.; WHEELW RIGHT, S. Restoring Our CompetitiveEdge: Competing Through Manufacturing. New York: John Wiley \& Sons, 1984.

IN MAN, R. Are you implementing a pull system by putting the cart before the horse? Production and Inventory Management Journal, v. 40, n. 2, p. $67-$ 71,1999 .

JAYARAMAN, V. Transportation, facility location and inventory issues in distribution network design. International Journal of O perations \& Production Management, v. 18, n. 5, p. 471-494, 1998. 
KWAN, A. The use of information technology to enhance supply chain management in the electronics and chemical industries. Production and Inventory Management Journal, v. 40, n. 3, p. 7-15, 1999.

LAMBERT, D. M.; STOCK, J. R.; ELLRAM, L. M. Fundamentals of Logistics Management. New York: Irwin-M CGraw Hill, 1998.

LANDVATER, D. World Class Production and Inventory Management. New York: Wiley, 1997.

$\mathrm{LI}, \mathrm{L}$. The role of inventory in delivery-time competition. Management Science, v. 38, n. 2, p. 182-198, 1992.

MENTZER, J. T.; KAHN, K. A framework of logistics research. Journal of Business Logistics, v. 16, n. 1, p. 231-250, 1995.

MENTZER, J.; KAHN, K.; BIENSTOCK, C. C. Sales Forecasting Executive Study. Knoxville: The University of Tennessee Press, 1996.

MOSER, C.; KALTON, G. Survey M ethods in Social Investigation. London: Heinemann Educational Books, 1971.

PAGH, J. D.; COOPER, M. C. Supply chain postponement and speculation strategies: how to choose the right strategy. Journal of Business Logistics, $v$. 19, n. 2, p. 13-33, 1998.

SILVER, E. A.; PETERSON, R. Decision Systems for Inventory M anagement and Production Planning. New York: Wiley \& Sons, 1985.
SIMCHI-LEVI, D.; KAMINSKY, P. D esigning and M anaging the Supply Chain: Concepts, Strategies and Case Studies. New York: McGraw-Hill, 2000.

STALK, G. Time: the next source of competitive advantage. Harvard Business Review, v. 66, n. 4, p. 41-51, 1988.

WANKE, P. Organização do fluxo de produtos como fase da estratégia logística de produtos acabados: uma síntese dos enfoques estático e dinâmico. Tese de Doutorado. Rio de Janeiro: COPPE/UFRJ, 2003.

WATERS, C.D.J. Inventory Control and Management. New York: Wiley \& Sons, 1992.

WEMMERLOV, U. Assemble-to-order manufacturing implications for materials management. Journal of Operations Management, v. 4, n. 4, p. 347-368, 1984.

ZINN, W.; BOWERSOX, D. Planning physical distribution with the principle of postponement. Journal of Business Logistics, v. 9, n. 2, p. 117136, 1988.

ZINN, W.; LEVY, M.; BOWERSOX, D. Measuring the effect of inventory centralization/decentralization on aggregate safety stock: "the square root law" revisited. Journal of Business Logistics, v. 10, n. 1, p. 1-13, 1989.

ZIPKIN, P. The limits of mass customization. MIT Sloan M anagement Review, v. 42, n. 3, p. 81-87, 2001.

\section{Artigo recebido em 17.12.2003. Aprovado em 28.07.2005.}

\section{Peter Fernandes Wanke}

Doutor em Ciências em Engenharia de Produção pela COPPEAD-UFRJ. Professor do Instituto Coppead de Administração da UFRJ e pesquisador do Centro de Estudos em Logística. Interesses de pesquisa nas áreas de planejamento da demanda, gestão de estoques em cadeias de suprimento e estratégia logística.

E-mail: peter@coppead.ufrj.br

Endereço: Ed. Coppead, $4^{\circ}$ andar, Cidade Universitária, Rio de Janeiro - RJ, 21949-900. 